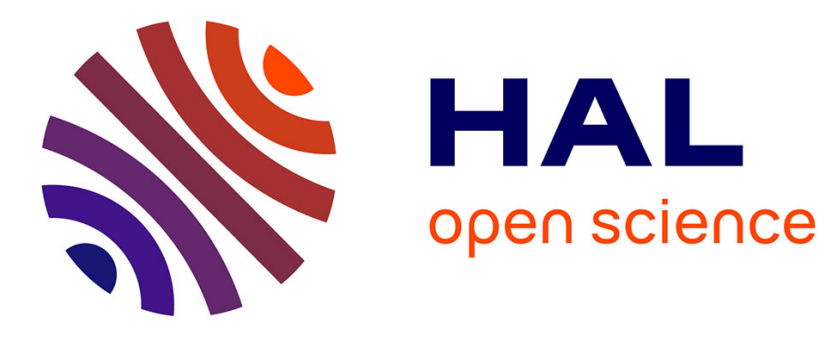

\title{
Control of a multi-degree of freedom worktool for vibrations assisted forging
}

Christophe Giraud-Audine, Thanh-Hung Nguyen, Michel Amberg, Betty Lemaire-Semail, Gabriel Abba, Régis Bigot

\section{- To cite this version:}

Christophe Giraud-Audine, Thanh-Hung Nguyen, Michel Amberg, Betty Lemaire-Semail, Gabriel Abba, et al.. Control of a multi-degree of freedom worktool for vibrations assisted forging. IEEE/ASME International Conference on Advanced Intelligent Mechatronics, AIM 2014, Jul 2014, Besançon, France. pp.812-817, 10.1109/AIM.2014.6878179 . hal-01109559

\section{HAL Id: hal-01109559 \\ https://hal.science/hal-01109559}

Submitted on 2 Feb 2015

HAL is a multi-disciplinary open access archive for the deposit and dissemination of scientific research documents, whether they are published or not. The documents may come from teaching and research institutions in France or abroad, or from public or private research centers.
L'archive ouverte pluridisciplinaire HAL, est destinée au dépôt et à la diffusion de documents scientifiques de niveau recherche, publiés ou non, émanant des établissements d'enseignement et de recherche français ou étrangers, des laboratoires publics ou privés. 


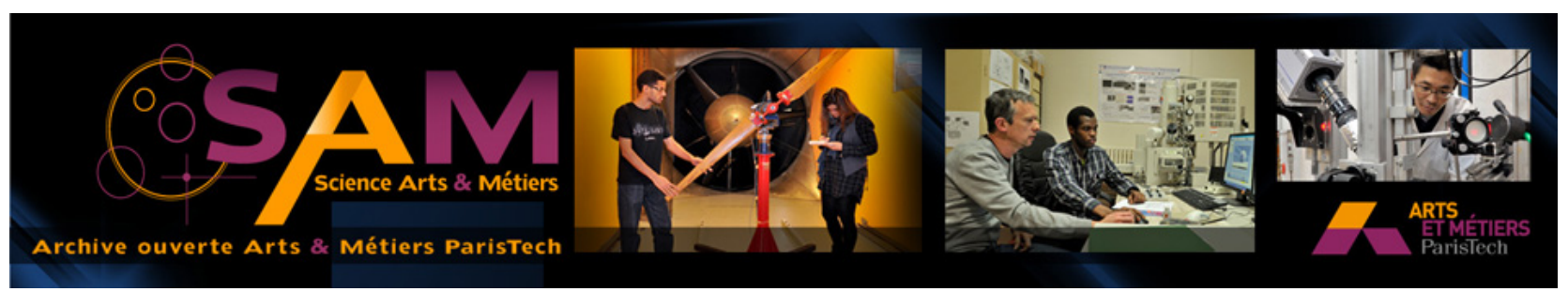

\section{Science Arts \& Métiers (SAM)}

is an open access repository that collects the work of Arts et Métiers ParisTech researchers and makes it freely available over the web where possible.

This is an author-deposited version published in: http://sam.ensam.eu

Handle ID: .http://hdl.handle.net/10985/9275

\section{To cite this version :}

Christophe GIRAUD-AUDINE, Thanh-hung NGUYEN, Gabriel ABBA, Régis BIGOT, Betty LEMAIRE-SEMAIL - Control of a multi-degree of freedom worktool for vibrations assisted forging In: IEEE/ASME International Conference on Advanced Intelligent Mechatronics, AIM 2014, France, 2014-07-08 - IEEE/ASME International Conference on Advanced Intelligent Mechatronics, AIM 2014 - 2014 


\title{
Control of a multi-degree of freedom worktool for vibrations assisted forging
}

\author{
C. Giraud-Audine ${ }^{1}$, T.H Nguyen ${ }^{1}$, M. Amberg ${ }^{3}$, B. Lemaire-Semail ${ }^{3}$ G. Abba $^{4}$ and R. Bigot ${ }^{2}$
}

\begin{abstract}
This paper addresses the control of a tool developed to superimpose vibrations along several directions on a piece during forging. Piezoelectric actuators are used to provide the required forces and speeds. Due to the minute displacements of the actuators, flexible hinges are used in a special arrangement in order realize the specified movements and to ensure controllability. The experimental results confirm the approach.

\section{INTRODUCTION}

In forging processes, a piece is forced into a shape given by dies. This requires huge forces which can be generated by an impact, or by moving steadily one of the dies. To reduce the necessary forces, the piece can be heated, or a lubricant can be used. Both solutions require supplementary operations, and energy. Vibrations have been known to influence material process during forming [1] or even cutting [2]. Some studies have demonstrated that applying ultrasonic vibrations superimposed to the movement of die can reduce the force, $\rightarrow$ similarly to the previous solutions [3], [4]. More recently, it was shown that low frequencies vibrations with special or combined waveforms can achieve the same reduction of the forging force, with less power required [5].

This work addresses the control of a special active tooling that generates vibrations in different directions by combining several piezoelectric actuators. According to preliminary studies [6], the tooling should be able to apply vibrations of arbitrary waveform along one direction while tilting the die around an arbitrary axis perpendicular to the first one to benefit from enhanced reduction of the effects.

First, the mechanical design and the specific power supply is described. Then the modelling is addressed. In the third part, using a systemic representation that encompasses the different subsystems of the tooling in a unified approach, the design the control structure is addressed. Finally, experimental results are presented in the last part.

\section{DESIGN}

\section{A. Mechanical Design}

For this application, the piezoelectric actuators have several advantages : high stiffness, large force, and high dynamics. However, the displacement range is limited to tens

${ }^{1}$ Laboratoire d'Electrotechnique et d'Electronique de Puissance (L2EP), Arts et Métiers Paristech, Centre de Lille, 8 bd Louis XIV, 59046 Lille, France christophe.giraud-audinedensam.eu

2 Laboratoire de Conception, Fabrication et Commande (LCFC), Arts et Métiers Paristech, Centre de Metz, 4 rue Augustin Fresnel, 57078 Metz Cedex 3, France

${ }^{3}$ L2EP, Université Lille 1, 59655 Villeneuve d'Ascq, France

${ }^{4}$ LCFC, Ecole Nationale d'Ingénieurs de Metz, Metz Technopole, 57000 Metz, France

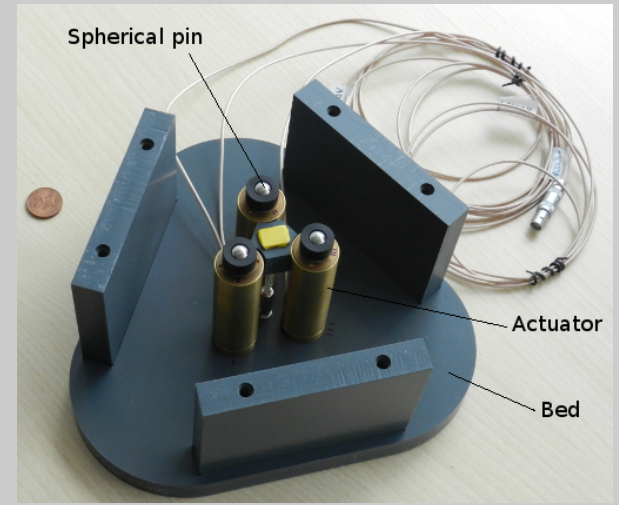

(a) Picture of the open tooling showing the actuator and the balls used to realize the spherical pin

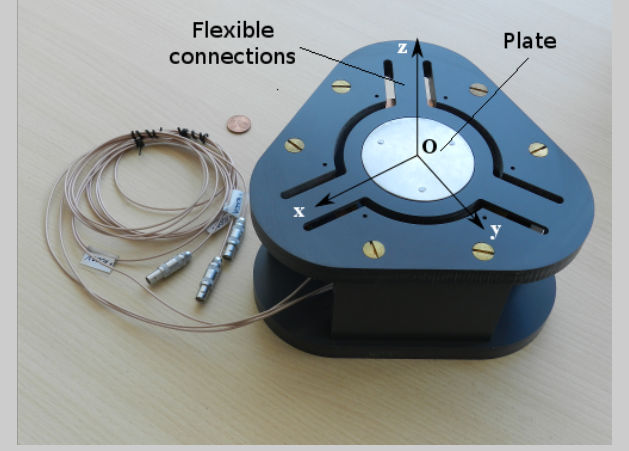

(b) Mounted tooling, the plate is supported by the actuators, flexible links are combined resulting in vertical displacement and two rotations out of the plane of the plate which will support the die.

Fig. 1: Vibrating tooling using three piezoelectric stack actuators

of $\mu \mathrm{m}$, therefore special attention must be paid during the design to avoid mechanical play. This motivates the use of flexure hinges combined in such ways that they realize the desired degrees of freedom (DOF) [7] or magnification [8]. During the design, attention must be paid to the rigidities that should be as small as possible along the desired DOF and very large for the other DOF, and the parasitic motions that may result from the imperfections of the hinges or from the kinematic are negligible [9].

The proposed mechanism (fig. 1) is based on three flexible links shifted by a $120^{\circ}$ along a circle in the plane of the tooling holding the die (plate cf. fig $1 \mathrm{~b}$ ). The main bending direction of the link being perpendicular to this plane this defines the translation DOF. The rotations are obtained thank to the torsions of the flexible links which, thanks to their 
special arrangement combine and give large compliances around two perpendicular directions within the plane of the die.

The plate is supported at three points by three piezoelectric stack actuators $^{1}$ (fig. 1a) which provide the necessary rigidity to support the forging force along the vertical axis, and that impose the attitude of the tooling plane thanks to punctual contact realized using spherical tips.

\section{B. Power Supply}

Piezoelectric devices have a very capacitive nature, resulting in large reactive currents. Several designs have been proposed for high power application, that take advantage of the resonance [10], [11]. However, the piezoelectric devices used her need some DC polarization which complicates the design [12].

For multiple actuators, resonant power supply is hindered by the disparities in capacitances and the need for nonsinusoidal waveforms. To avoid the use of a transformer, the power supply is realized using an off-the-shelve inverter, a half bridge rectifier is connected directly to the piezoelectric actuators (fig 2). This configuration take advantages of the current reversibility of the inverter to realize a boost DC to DC conversion in order to charge the bus capacitor. To do so, the duty ratios of the inverter are set to a constant value generating a lower voltage than the DC supply voltage. The achieved. other displacements and therefore not considered. With those assumptions, the plane position is entirely defined by the pin positions. A straightforward calculation gives the relationship between the tip speed and the speeds at the center of the plate $O$ :

$$
\underbrace{\left[\begin{array}{l}
v_{p_{1}} \\
v_{p_{2}} \\
v_{p_{3}}
\end{array}\right]}_{v_{p}}=\underbrace{\left[\begin{array}{cccccc}
0 & 0 & 1 & 1 & 1 & 0 \\
0 & 0 & R & -\frac{R}{2} & \frac{-\sqrt{3} R}{2} & 0 \\
0 & 0 & 0 & -\frac{R}{2} & -\frac{\sqrt{3} R}{2} & 0
\end{array}\right]}_{\mathbf{A}} \underbrace{\left[\begin{array}{c}
v_{x} \\
v_{y} \\
v_{z} \\
\omega_{x} \\
\omega_{y} \\
\omega_{z}
\end{array}\right]}_{\mathbf{v} O}
$$

where $v_{k}$ and $\omega_{k} k \in x, y, z$ denote the translational and rotational speeds ${ }^{2}$ measured at the center $O$ within the reference frame, while $v_{1}, v_{2}$ and $v_{3}$ are the translational speeds along $z$ at the contact points $C_{1}, C_{2}$ and $C_{3}$, which are located on a circle (radius $R$, center $O$ ). Moreover, considering that the friction is small compared to the normal load, the generalized forces at the end of the tip can be written as follow :

$$
\mathbf{F}_{\mathbf{S i}}=\left[0,0, F_{i z}, 0,0,0\right]^{\top}
$$

Thus, following the screw theory, the components of the generalized forces and moment at point $O$ are :

$$
\mathbf{F}_{\mathbf{O}}=\underbrace{\left[\begin{array}{ccc}
0 & 0 & 0 \\
0 & 0 & 0 \\
1 & R & 0 \\
1 & -\frac{R}{2} & -\frac{\sqrt{3} R}{2} \\
1 & -\frac{R}{2} & \frac{\sqrt{3} R}{2} \\
0 & 0 & 0
\end{array}\right]}_{\mathbf{A}^{\top}} \underbrace{\left[\begin{array}{c}
F_{1 z} \\
F_{2 z} \\
F_{3 z}
\end{array}\right]}_{f_{p}}
$$

When the duty ratios $m_{k}, k \in 1,2,3$ are varied and maintained at values such that is greater than the DC voltage $U_{d c}$, the diodes stop conducting, and the inverter operations take place. The two modes can be applied alternatively, that is inverter operations are switched off to charge the DC bus capacitor when it reaches a minimum value, then switched back once the desired DC voltage is obtained, or can be mixed [13].

\section{DYNAMIC MODEL}

\section{A. Kinematic}

As already explained, the tooling plate motion is imposed by the actuator using spherical pins. This solution is only able to push against the piece, although this is not a real limitation because the forging force is very large, and press the plate against the spherical pins. The diameters of the sphere are calculated in order to transmit $5500 \mathrm{~N}$ without noticeable deformations compared to the $40 \mu \mathrm{m}$ stroke of the piezoelectric actuators. Thus, to model the kinematic, it is assumed that the plate remains in contact with the tips, and that their deformations are negligible. Besides, considering the compliances of the guiding (see III-C), displacements in the $x$ and $y$ directions and rotation around the $z$ axis (cf. 1b) are second order of magnitude compared to the

${ }^{1}$ Piezomechanik HPSt 1000/15-8/60 : maximum force $5500 \mathrm{kN}$,maximum displacement $80 \mu \mathrm{m}$ for a $-200 \mathrm{~V} / 1000 \mathrm{~V}$ supply voltage
The power of the actuators is calculated by :

$$
\mathcal{P}=f_{p}^{\top} v_{p}=f_{p}^{\top} A \mathbf{v}_{O}=\left(A^{\top} f_{p}^{\top}\right)^{\top} \mathbf{v}_{O}=\mathbf{F}_{\mathbf{O}} \mathbf{v}_{O}
$$

\section{B. Power Supply}

The frequency range of the device is below $100 \mathrm{hz}$. The actuators can therefore be modelled considering the quasistatic piezoelectric equation. For piezo-actuator $k$, considering fixed-free mechanical operation, the tip displacement $w_{p_{k}}=\int v_{p_{k}} d t$ and the total electrical charge $Q_{p_{k}}=\int i_{p_{k}} d t$ are linear functions of the actuator voltage $u_{p_{k}}$ and the force $F_{z_{k}}$ applied by the actuator as follows :

$$
\begin{aligned}
F_{z_{k}} & =-k_{s} w_{p_{k}}+N u_{p_{k}} \\
Q_{p_{k}} & =N w_{p_{k}}+C_{p} u_{p_{k}}
\end{aligned}
$$

where $k_{s}$ is the short-circuited rigidity of the actuator, $N$ is the force factor resulting from the piezoelectric conversion and $C_{p}$ is the free capacitance of the actuator. Deriving (5b) with respect to time, one can see that the current in

\footnotetext{
${ }^{2}$ In the following, we will refer to the generalized speeds at a point defined as the vector gathering the translational $(V)$ and rotational speeds $(\Omega)$ at this point : $\mathbf{v}=\left[V^{\top} \Omega^{\top}\right]^{\top}$. Similarly, generalized forces concatenate the forces $F$ and moments $M$ at the considered point : $\mathbf{F}=\left[F^{\top} M^{\top}\right]^{\top}$
} 


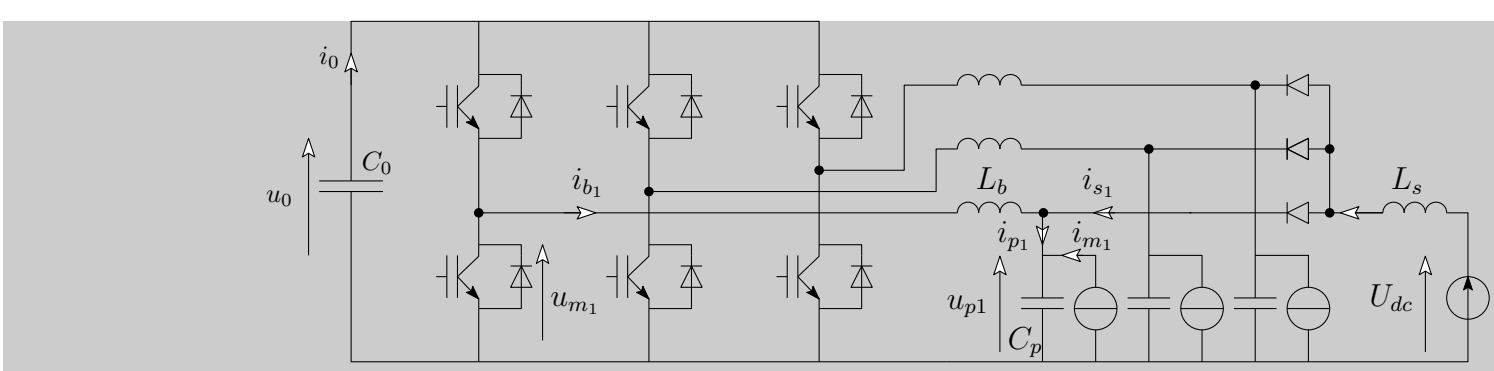

Fig. 2: schematic of the power supply

the piezoactuator has two contributions. One is due to the dielectric through the free capacitance, the second is a current source describing the charges transfer inside the piezoelectric material due to the strain. The later is called the motional current. For quasistatic operations it is small compared to the reactive current due to $C_{p}$.

Considering the case when $u_{p_{k}}>U_{D C}$, the diodes are non conducting. The inverter switches are controlled by a pulse width modulation (PWM) with a $30 \mathrm{kHz}$ carrier. Considering the $3 \mathrm{kHz}$ cut-off frequency of the $L_{b}-C_{p}$ branch, a mean model of the inverter is considered :

$$
\left\{\begin{aligned}
i_{0}(t) & =\sum_{k=1}^{3} m_{k}(t) i_{b_{k}}(t) \\
u_{m_{k}}(t) & =m_{k}(t) u_{0}
\end{aligned}\right.
$$

The voltage of piezoactuator $k$ is then :

$$
L_{b} C_{p} \frac{d^{2} u_{p_{k}}}{d t^{2}}+u_{p_{k}}=m_{k} u_{0}+L_{b} \frac{d i_{m k}}{d t} \quad(k \in 1,2,3)
$$

\section{Dynamic of the Flexible Guiding}

The flexible hinges can considered as thin rods. Using the classical beam theory, it is then possible to relate the displacement of the tips of the hinges to the mechanical load at the tip ends [14]. The elasticity matrix at the center of the plate $\mathbf{O}$ is calculated according to the screw theory [15]. In the reference frame of fig. $1 \mathrm{~b}$, it writes :

$$
\mathbf{K}=\left[\begin{array}{cccccc}
k_{1} & 0 & 0 & 0 & -k_{2} & 0 \\
0 & k_{1} & 0 & k_{2} & 0 & 0 \\
0 & 0 & k_{5} & 0 & 0 & 0 \\
0 & k_{2} & 0 & k_{3} & 0 & 0 \\
-k_{2} & 0 & 0 & 0 & k_{3} & 0 \\
0 & 0 & 0 & 0 & 0 & k_{4}
\end{array}\right]
$$

The various rigidity can be expressed from the geometry and the material properties of the hinges and are not detailed here. For the matter of interest in the scope of this paper it suffices to know that by construction : $k_{1} \gg k_{5} \quad k_{3} \gg k_{4}$ . The inertia matrix at the same point can be easily deduced from the inertia matrix of a cylinder using the parallel axis theorem :

$$
\mathbf{M}=\left[\begin{array}{cccccc}
m_{1} & 0 & 0 & 0 & -m_{2} & 0 \\
0 & m_{1} & 0 & m_{2} & 0 & 0 \\
0 & 0 & m_{1} & 0 & 0 & 0 \\
0 & m_{2} & 0 & m_{3} & 0 & 0 \\
-m_{2} & 0 & 0 & 0 & m_{3} & 0 \\
0 & 0 & 0 & 0 & 0 & m_{4}
\end{array}\right]
$$

Assuming small displacement of the center of the plate , the motion equations are thus :

$$
\mathbf{M} \ddot{\mathbf{w}}+\mathbf{K w}=\mathbf{F}_{O}+\mathbf{F}_{l}
$$

where $\mathbf{w}=\left[w_{x}, w_{y}, w_{z}, \alpha_{x}, \alpha_{y}, \alpha_{z}\right]^{\top}$ is the generalized displacement of the center $\mathbf{O}$ of the plate, and $\mathbf{F}_{l}=$ $\left[0,0, F_{l}, M_{l x}, M_{l y}, 0\right]^{\top}$ is the generalized force vector applied by the forged part onto the plate.

It follows from the structure of the matrices that the tooling dynamic consists in four independent dynamic subsystems :

$$
\begin{gathered}
\Sigma_{1}:\left[\begin{array}{cc}
m_{1} & -m_{2} \\
-m_{2} & m_{3}
\end{array}\right]\left[\begin{array}{c}
\ddot{w}_{x} \\
\ddot{\alpha}_{y}
\end{array}\right]+\left[\begin{array}{cc}
k_{1} & -k_{2} \\
-k_{2} & k_{3}
\end{array}\right]\left[\begin{array}{l}
w_{x} \\
\alpha_{y}
\end{array}\right] \\
=\left[\begin{array}{c}
0 \\
M_{y}+M_{l y}
\end{array}\right] \\
\Sigma_{2}:\left[\begin{array}{ll}
m_{1} & m_{2} \\
m_{2} & m_{3}
\end{array}\right]\left[\begin{array}{c}
\ddot{w}_{y} \\
\ddot{\alpha}_{x}
\end{array}\right]+\left[\begin{array}{cc}
k_{1} & k_{2} \\
k_{2} & k_{3}
\end{array}\right]\left[\begin{array}{c}
w_{y} \\
\alpha_{x}
\end{array}\right] \\
=\left[\begin{array}{c}
0 \\
M_{x}+M_{w l x}
\end{array}\right] \\
\Sigma_{3}: m_{1} \ddot{w}_{z}+k_{5} w_{z}=F_{z}+F_{l z} \\
\Sigma_{4}: m_{4} \ddot{\alpha}_{z}+k_{5} \alpha_{z}=0
\end{gathered}
$$

$\Sigma_{3}$ is the translational dynamic along the direction $O_{z}$, while $\Sigma_{4}$ is the rotational dynamic around the same axis. The later is not controllable, while $\Sigma_{3}$ is controlled by the mean force applied by the actuators.

Systems $\Sigma_{1}$ and $\Sigma_{2}$ are similar and show the cross coupling between rotation around the axis $O x$ (resp. $O y$ ) with displacement $O y$ (resp. $O x$ ). This results from the design where the flexible hinge neutral plane are not coincident with the orthocenter of the triangle defined by the contact points of the sphere with the plate.

\section{Simplified Dynamic}

In this section, the models of $\Sigma_{1}, \Sigma_{2}$ are further simplified considering the different dynamics involved. Using a modal decomposition, each subsystem can be transformed in a new basis resulting in the new systems $\Sigma_{i}^{\prime}, i \in\{1,2\}$ where the new state vectors have independent components. In this part, the discussion is based on $\Sigma_{1}$, and can be straightforwardly applied to $\Sigma_{2}$. The new system writes :

$$
\left[\begin{array}{l}
\ddot{x}_{1} \\
\ddot{x}_{2}
\end{array}\right]+\left[\begin{array}{cc}
\lambda_{1} & 0 \\
0 & \lambda_{2}
\end{array}\right]\left[\begin{array}{l}
x_{1} \\
x_{2}
\end{array}\right]=\left[\begin{array}{l}
\tau_{1} \\
\tau_{2}
\end{array}\right]
$$

where $\lambda_{1}, \lambda_{2}$ are the eigenvalues of the matrix $\mathbf{G}=$ $\left[\begin{array}{cc}m_{1} & -m_{2} \\ -m_{2} & m_{3}\end{array}\right]^{-1}\left[\begin{array}{cc}k_{1} & -k_{2} \\ -k_{2} & k_{3}\end{array}\right]$ and $\left[\begin{array}{c}x \\ \alpha_{y}\end{array}\right]=\mathbf{P}\left[\begin{array}{l}x_{1} \\ x_{2}\end{array}\right] \mathbf{P}$ being a 
matrix whose column are the eigenvectors. The input vector in the eigen basis is calculated by :

$$
\left[\begin{array}{l}
\tau_{1} \\
\tau_{2}
\end{array}\right]=\mathbf{P}^{-1}\left[\begin{array}{cc}
m_{1} & -m_{2} \\
-m_{2} & m_{3}
\end{array}\right]^{-1}\left[\begin{array}{c}
0 \\
M_{y}+M_{l y}
\end{array}\right]
$$

Hence, its component are proportional to $M_{y}-M_{l y}$. As already mentionned, the translational rigidities are larger by several orders of magnitude compared to the rotational rigidities. As a consequence, $\lambda_{1} \gg \lambda_{2}$ meaning that $x_{1}$ will have reached steady state much faster than $x_{2}$ the later being the so-called dominant mode. Replacing this assumption in 15 :

$$
\left\{\begin{array}{l}
0=-\lambda_{1} x_{1}+\tau_{1} \\
\ddot{x_{2}}=-\lambda_{2} x_{2}+\tau_{2}
\end{array}\right.
$$

gives after some algebra, and casting the system back into the original coordinates, the system is reduced to :

$A\left(m_{3}-\frac{p_{12}}{p_{22}} m_{2}\right) \ddot{\alpha}_{y}+A\left(k_{3}-\frac{p_{12}}{p_{22}} k_{2}\right) \alpha_{y}=M_{y}-M_{l y}$

with $A=\frac{1}{1+k_{2} \frac{p_{22} m_{2}-p_{12} m_{1}}{\lambda_{1} p_{22}\left(m_{1} m_{3}-m_{2}^{2}\right)}}$, and $p_{k l}, k, l \in 1,2$ are the components of $\mathbf{P}$.

\section{CONTROL}

\section{$\rightarrow$ A. Macroscopic Energetic Representation}

In order to design the control structure, the Energetic Mascrocopic Representation (EMR) is used [16]. The EMR is a graphic representation, based on the equations of a system that highlights the power flows within a system. It relies on the fact that a subsystem composing a larger system interact with the others by reacting to the action it is submitted to. An important piece of information that is implicitly conveyed by this concept is causality which derives from the energy storages in the system.

The worktool's EMR is depicted on fig 3 (orange blocks and green blocks). The diodes are supposed to be off because the strategy used consists in controlling the charge of the DC bus and the vibrations control as two separate phases. The energy storages, which states are defined by the integration of action variables imposed by other components, are represented by crossed box. Other blocks connect different component through constraints (e.g node currents or forces equilibrium), or represent conversion (e.g the piezoelectric conversion). Finally, sources can also be used to represent external interaction with the system (e.g voltage or force sources).

The benefit of using the EMR is that the control structure can be systematically deduced by inversion of the gain in the system, and placing controllers to approximate the inverse of an energy storage. Applying the method to the system results in the maximal control structure, in the sense that it assumes that all the necessary variables are measured. The second outcome is that by subdividing the control to the control of the energy storage, physical limitations are easier to respect when designing the controller. Then, for obvious practical

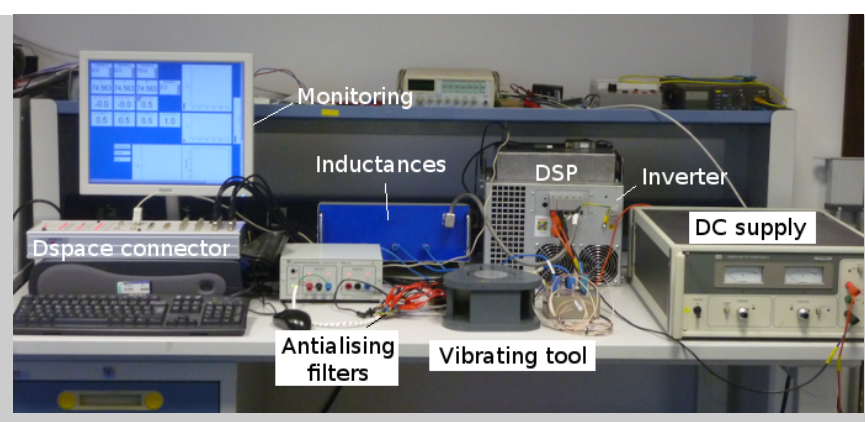

Fig. 4: Experimental set-up

reasons, the designer task consists in simplifying the control structure, especially with regards to the measure availability, and design the controller accordingly.

Following this approach, the resulting control structure is a cascaded control (fig 3 blue blocks), successively controlling the current supplied to the actuator, their voltage to finally achieve the desired speeds. The maximal control structure uses direct compensation (dashed arrows) to account for the constraint of the system (here to compensate the elasticities). For practical considerations, they are not implemented in this work although they could be estimated. Another simplification adopted in the experiment, was to by-pass the current control loop to avoid the difficulty of the current measurement which are particularly noisy (due to the capacitive nature of the load)s.

\section{EXPERIMENTAL RESULTS}

\section{A. Experimental Set-up}

The set-up developed in order to validate the control is presented on fig 4. the inverter is a semikron $(1200 \mathrm{~V}, 60 \mathrm{~A})$, the PWM is generated thanks to a EzDSP TMS320F2812 with a carrier frequency of $30 \mathrm{kHz}$. The duty ratio are transmitted through RS232. The communication maximum speed is 19200 bauds, and the length of the messages for a three-phase modulation requires 9 ascii characters resulting in a limited sampling time of $1201 \mathrm{~Hz}$. Therefore, to avoid excitation of modes of the mechanical set-up, the transmitted duty ratio $m_{k}$ are interpolated using a first order hold. As a result, the duty ratio are updated at $30 \mathrm{kHz}$ internally by the DSP, far above the mechanical resonance. The $10 \mathrm{mH}$ inductances $L_{b}$ were chosen according to the theoretical capacitance of the piezo-actuators of $270 \mathrm{nF}$ so as to have a cut-off frequency of ca $3 \mathrm{kHz}$.

The control being limited at $1201 \mathrm{~Hz}$, all measurement are filtered $(600 \mathrm{~Hz}$ second order butterworth low-path filter) to avoid aliasing. For the electrical side, the voltages $u_{0}$, $u_{p_{k}}$ are measured. The vertical displacement $w_{z}$, and the angles are calculated thanks to three vertical displacement measurements on the outer diameter of the plate regularly spaced $120^{\circ}$ opposite to each hinge. To do so, magnet were fixed to the plate and hall sensors fixed to the tooling's bed were used.

The control is executed by a DSPace 1104 board programmed using Simulink. It also provides a mean to define 


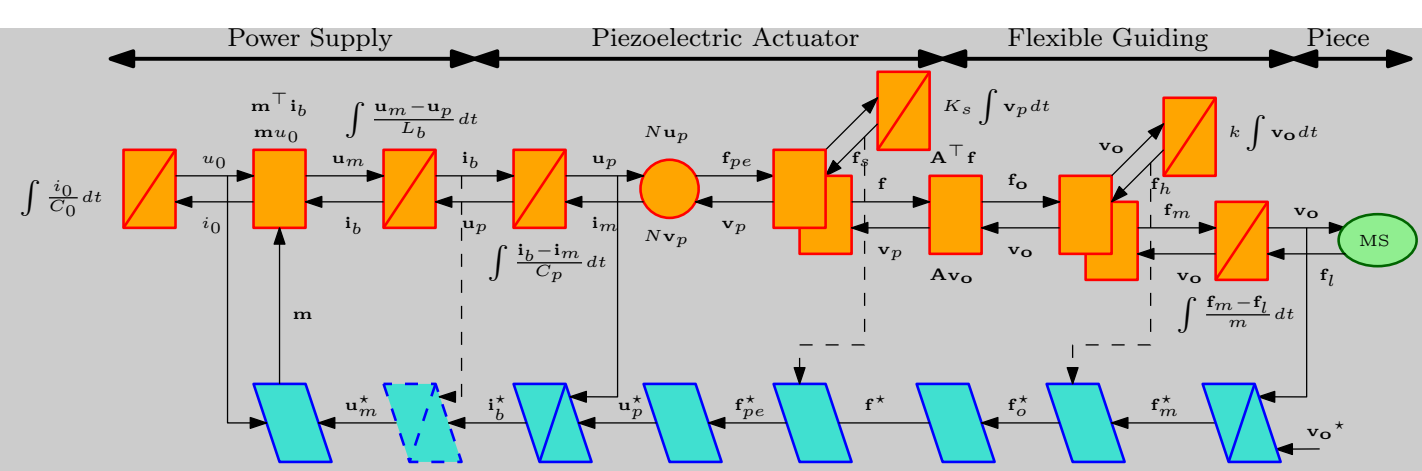

Fig. 3: EMR of the system (orange and green blocks refer to the system, blue to the control).

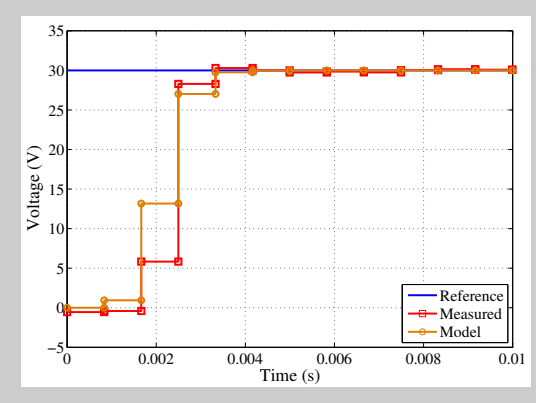

(a) Comparison of the model and measured response to a voltage step.

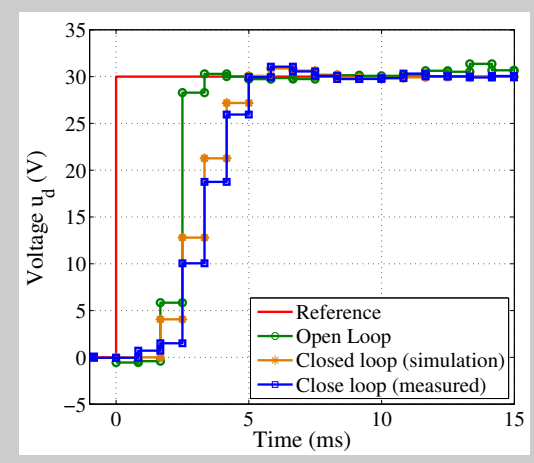

(b) Closed Loop responses.

Fig. 5: Voltages control

interfaces for adjusting gains or measure and visualise different variables of the process.

\section{B. Voltages control}

The main purpose of the flexible guiding rotations is to generate a rotating tilt of the plate that is superimposed to the vertical vibrations. It can be shown (this point will not be discussed in this paper for conciseness) that such a motion is obtained by applying three phased sinusoidal voltages. That follows from the design which is similar to magnetic alternative machines, and is somewhat visible from the matrix $\mathbf{A}$ (1). As a consequence, the control of voltages is performed in a rotating frame $d, q, h$ [17]. The $d q$ components of the voltage control amplitude of the tilting, while the homopolar component generates the vertical waveform. In the process of transforming (7) into the rotating frame cross coupling appears, but have little influence in the speed range of interest. On fig. 5a, the step response of the transfer function resulting from the model (including one order hold and antialiasing filter) with coupling neglected is compared to the measured one, and are in good agreement. It also validates the decoupling of the electrical and mechanical dynamics (through the motional current) since the later is not taken into account to calculate the transfer function.

Based on this model, a proportional integral corrector is calculated. Fig. 6b compares open-loop step response with the theoretical and the actual response in closed loop on axis $d$ (comparable results are obtained on the other axis by symmetry of the design). Good agreement of the closed loop is found, and validate the design. The control also prevents $\leftarrow$ fluctuating voltage such as can be seen for open-loop at $t=14 \mathrm{~s}$ (fig. 5b).

\section{Motion control}

The purpose of the outer loop is to compensate for the imperfections and the disturbances such as the disparity of the actuators, the uncertainties on their locations and on the dimensions of flexible links. Besides, non-linearities of the piezoelectric materials will change the piezoelectric coefficient as the mechanical stress increases during forging [18].

It can be noticed from (11), (12) and (13) that the mechanical dynamic is already in a $\alpha, \beta$ frame. A rotation transformation casts the speeds in the same $d q h$ frame of the voltages. Thus, to obtain a rotating tilt of the plate, the speed $v_{d}, v_{q}$ should be constant. In practice, since we are only interested in the rotating speed, $v_{q}$ reference is set to zero, and $v_{d}$ directly imposes the desired speed. Pi controllers can be used.

$v_{h}$, the homopolar speed controls the vertical vibrations speed. The required waveforms are not limited to sine wave, but can be triangle, gaussian impulses etc... Moreover, the piezoelectric actuator will be compressed at (roughly) constant speed under the forging force. The control should not attempt to compensate this because the voltage would rapidly increase until saturation to maintain the mean position of the tip of the actuators. Indeed, looking at (5a), it can be seen that to keep $w_{p k}$ while $F_{p k}$ increases, implies a proportional rise of the voltage. Therefore, the control is applied on the $d q$ axis, while the homopolar axis $h$ remains in open loop. On fig. 6 the open and closed loop response of $v_{p d}, v_{p q}$ 


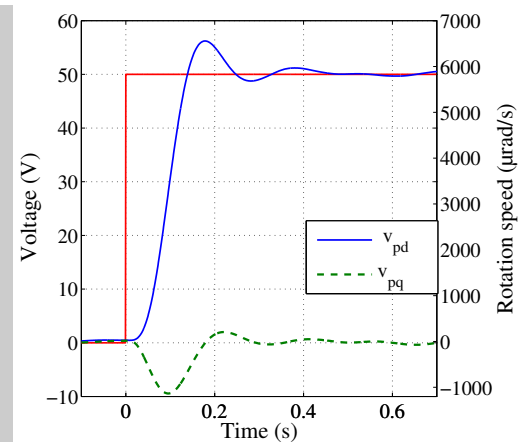

(a) Open loop response in the $d q$ frame.

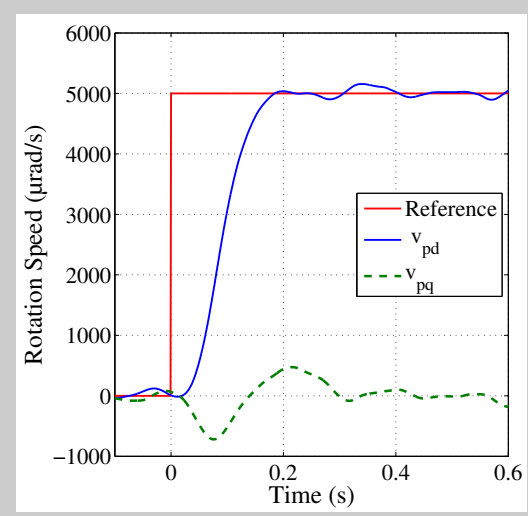

(b) Closed loop response.

Fig. 6: Speeds Control

$54 \mathrm{pt}$ 0.75 in $19.1 \mathrm{~mm}$ are compared. The speed are estimated by derivating the positions and then filtered in the $d q$ frame. The control reduces the overshoot and the settling time. Some variations around the reference remain because the homopolar speed is not controlled.

\section{CONCLUSION}

This paper has presented an original design for a worktool used in forging process. The components of this mechatronic systems have been explained, and models have been proposed. To design the control, the system is represented using a mutiphysic representation tool that respects causality and depicts the power transfer in the system. A control structure has been proposed, and simple controllers calculated. An experimental set-up has been realised, and the tests have confirmed the expected results.

\section{REFERENCES}

[1] F. Blaha and B. Langenecker, Plastizittsuntersuchungen von metallkristallen in ultraschallfeld, Acta Metallurgica, vol. 7, no. 2, pp. 93100, Feb. 1959. (in german)
[2] V. I. Babitsky, A. N. Kalashnikov, A. Meadows, and A. Wijesundara, Ultrasonically assisted turning of aviation materials, Journal of materials processing technology, vol. 132, no. 1, pp. 157-167, 2003.

[3] K. Siegert and A. Mck, Wire drawing with ultrasonically oscillating dies, Journal of Materials Processing Technology, vol. 60, no. 1-4, pp. 657-660, Jun. 1996.

[4] Z. Huang, M. Lucas, and M. J. Adams, Influence of ultrasonics on upsetting of a model paste, Ultrasonics, vol. 40, no. 1-8, pp. 43-48, May 2002.

[5] R. Ly, C. Giraud-Audine, G. Abba, and R. Bigot, Experimentally valided approach for the simulation of the forging process using mechanical vibration, International Journal of Material Forming, vol. 2, no. S1, pp. 133-136, Dec. 2009.

[6] A. Khan, C. Giraud-Audine , G. Abba, R. Bigot, Effects of Vibrations on Metal Forming Process: Analytical Approach and Finite Element Simulations, International Conference on Avances in Materials and Processing Technologies (AMPT2010). AIP Conference Proceedings, Volume 1315, 2011, pp. 787-792.

[7] Jae W. Ryu, Dae-Gab Gweon, Kee S. Moon, Optimal design of a flexure hinge based XY $\theta$ wafer stage, Precision Engineering, Volume 21, Issue 1 , July 1997 , pp. 18-28.

[8] S. B. Choi, S. S. Han, Y. M. Han, and B. S. Thompson, A magnification device for precision mechanisms featuring piezoactuators and flexure hinges: Design and experimental validation, Mechanism and Machine Theory, vol. 42, no. 9, pp. 1184-1198, Sep. 2007.

[9] Tim King, Wei Xu, The design and characteristics of piezomotors using flexure-hinged displacement amplifiers, Robotics and Autonomous Systems, Volume 19, Issue 2, December 1996, Pages 189-197.

[10] R. Li, M. Loenneker, N. Froehleke, and J. Boecker, Design of Power Supply for Driving High Power Piezoelectric Actuators, in Industry Applications Society Annual Meeting, 2008. IAS'08. IEEE, 2008, pp. $1-6$.

[11] D. H. Tran, J.-F. Rouchon, B. Nogarede, and C. Viguier, Design a power inverter and transformer for piezoelectric actuator, in Electronics, Control, Measurement and Signals (ECMS), 2011 10th International Workshop on, 2011, pp. 1-6.

[12] E. Goenaga and J.-P. Ferrieux, Contactless Power Transfer System for High Power Piezoelectric Actuators in Aeronautical Applications, presented at the APEC Conference, Long Beach, California, 2013.

13] C. Giraud-Audine, M. Amberg. F. Giraud, and B. Semail, A simplified power supply for piezoelectric actuators used in forging processes, in Power Electronics and Applications (EPE), 2013 15th European Conference on, 2013, pp. 1-9.

[14] N. Ciblak and H. Lipkin, Remote center of compliance reconsidered, in Proceedings of The 1996 ASME Design Engineering Technical Conference and Computers in Engineering Conference, Irvine, 1996 pp. 1-10.

[15] W. Khalil, Modeling, identification and control of robots. London Sterling, VA: Kogan Page Science, 2004.

[16] A. Bouscayrol, B. Davat, B. de Fornel, B. Franois, J. P. Hautier, F. Meibody-Tabar, and M. Pietrzak-David, Multi-converter multimachine systems: application for electromechanical drives, The European Physical Journal Applied Physics, vol. 10, no. 2, pp. 131-147, May 2000.

[17] J.-P. Louis, Ed., Control of non-conventional synchronous motors. London Hoboken, NJ: ISTE Wiley, 2012.

[18] G. Yang, S.-F. Liu, W. Ren, and B. K. Mukherjee, Uniaxial stress dependence of the piezoelectric properties of lead zirconate titanate ceramics, in SPIE's 7th Annual International Symposium on Smart Structures and Materials, 2000, pp. 103-113. 